www.jmscr.igmpublication.org Impact Factor 5.244

Index Copernicus Value: 83.27 ISSN (e)-2347-176x ISSN (p) 2455-0450 crossref DOI: _https://dx.doi.org/10.18535/jmscr/v4i11.12

\title{
Comparison of Rapid Fungal Staining Technique 1\% Chicago Blue 6B stain for the Diagnosis of Superficial Fungal Infections (Pityriasis Versicolor/ Tinea Versicolor) with 10\% KOH Mount and Routine Fungal Culture on Sabouraud's Dextrose Agar with Olive Oil Overlay
}

\author{
Authors \\ N. Kamath ${ }^{1}$, R. Swaminathan', Jyoti Sonawane ${ }^{3}$, Nadeem Amin ${ }^{4}$ \\ ${ }^{1}$ Assistant Professor, Dept. of Microbiology, D Y Patil University School of Medicine, Nerul \\ ${ }^{2}$ Professor \& H.O.D., Dept. of Microbiology, D Y Patil University School of Medicine, Nerul \\ ${ }^{3}$ Assistant Professor, Dept. of Microbiology, D Y Patil University School of Medicine, Nerul \\ ${ }^{4}$ Senior resident, Dept. of Microbiology, D Y Patil University School of Medicine, Nerul \\ Corresponding Author \\ Dr N. Kamath \\ 601, Aston Tower, Plot No.9B, Sector 20.Kharghar, Navi Mumbai, 410210 \\ Email: nktallur@gmail.com
}

\begin{abstract}
Pityriasis versicolor is a common skin infection all over the world caused by Malassezia furfur. 10\% KOH mount is done for all clinically suspected Tinea versicolor by taking skin scrapings. $10 \% \mathrm{KOH}$ has shown $66.66 \%$ positives in this study. When we add 1\% Chicago Blue 6B stain it enhances the results to $93.33 \%$ which is as good as the Gold standard that is culture on SDA with a overlay of sterile Olive Oil. Culture has shown $96.66 \%$ positivity as Malassezia furfur grows well when there is oil overlay. So $1 \%$ Chicago Blue $6 B$ stain can be routinely incorporated in Mycology laboratories and also in Skin clinics as the procedure is simple, rapid, reliable and can be identified with a good contrast and specificity/sesitivity is as good as culture. $1 \%$ Chicago Blue $6 \mathrm{~B}$ is more efficacious than usual 10\% KOH mount in diagnosing superficial fungal infections caused by Malassezia furfur.

KeyWords: 1\% Chicago Blue stain(CSB), Malassezia furfur, 10\% KOH, SDA with Chloramphenicol and cycloheximde.
\end{abstract}

\section{Introduction}

Malassezia furfur is a yeast which causes Pityriasis versicolor (Tinea versicolor) which is a Superficial (cutaneous) fungal infection. Clinically lesions are characterized by scaly macules or patches. Their color varies from red, pale yellow, brown, to dark brown. It is generally explained as hypopigmented patches on the skin. M.furfur is a small $(1.5-4.5 \mu \mathrm{m})$ yeast which is with bottle shaped cells buds separated from parent cell by a septum. Hyphae are 2.5 to $4 \mu \mathrm{m}$ in size. Hyphae are short curved fungal elements. Infection can occur on the trunk, arms, shoulders and face. P.versicolor is worldwide in distribution but most common in tropical countries.

Patient population includes farmers, field workers, carpenters and people who work in sunlight for a long period of time and people who sweat a lot. 
These were the common predisposing factors seen in these patients.

All the skin scrapings are subjected to $10 \% \mathrm{KOH}$ mount, 1\% Chicago Blue Mount 6B mount and Culture on Sabouraud's Dextrose agar with sterile olive oil overlay as M.furfur is known to be lipophilic. $1 \%$ Chicago Blue stain $6 \mathrm{~B}$ is superior than $\mathrm{KOH}$ wet mount. This has been shown by studying P.versicolor and Dermatophytes ${ }^{1}$. When a comparison was made between Chicago blue, Chlorazole black, and Parker ink for the rapid diagnosis of skin and nail infections, study proved that Chicago Blue-KOH provides a good color contrast $^{2,4,5}$. It is new, superior to other techniques. However other techniques like Parker Ink with $\mathrm{KOH}$, Per-iodic Acid Schiff, Calcoflour white, Lacto phenol cotton Blue, PCRs, are available but there are limitations like expertise, sensitivity, specificity and cost factors too.

\section{Materials and Methods}

All the clinically suspected patients from Skin outpatient Department which included male and female patients of D Y Patil Hospital, Nerul, Navi Mumbai. Areas affected with suspected fungal infection (Pic.1) were cleaned with alcohol swabs to remove bacterial contamination.

Adequate amount of 120 Skin Scrapings from patients with clinically suspected cases of Pityriasis versicolor were collected and made into three parts. The skin scrapings were taken by using blunt side of 15 number sterile surgical blades. One part was for $10 \% \mathrm{KOH}$ mount, second part was for $1 \%$ Chicago Blue $6 \mathrm{~B}$ and the third part was for culture on SDA.

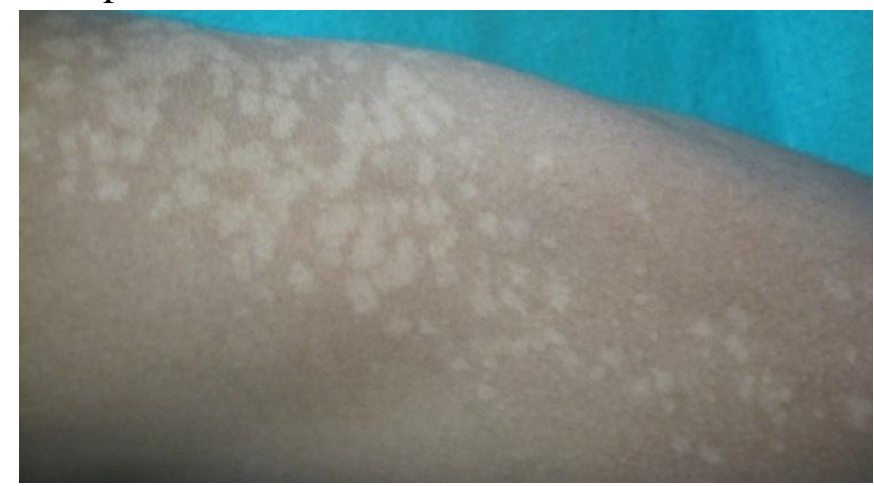

Pic .1: Common clinical picture.(Hypo pigmented chronic, recurrent lesions)

\section{Microscopy}

This is a direct microscopic examination of the above specimen to detect fungal spores or hyphae. Initial examination is with low power magnification (x10) and low intensity of light with lowering of the condenser. Later, for a higher magnification (x40), the condenser should be higher for better illumination to study the morphology of the fungus. Fungal spores vary from $2-10 \mu \mathrm{m}$ in diameter. Wet mounts were examined in $10 \mathrm{X}$ and $40 \mathrm{X}$ with the $10 \% \mathrm{KOH}$ and the $1 \%$ CSB stains. (Mix 1 drop of CSB and 1 drop of $10 \% \mathrm{KOH}$ and dropped on the slide with skin scrapings). The slides were observed immediately and which were negative for fungal elements were kept for up to 30 minutes along with moistened sterile filter paper in a sterile Petri dish in a humidifying chamber. Typical spaghetti and meat ball appearance (Pics.2,3,4) was observed which is a characteristic feature of Malassezia furfur.

\section{Culture}

As per the plan of the study third part of the skin scrapings were cultured on Sabouraud's Dextrose $\operatorname{agar}^{3} \quad$ with Chloramphenicol $(0.05 \%)$ and Cycloheximide $(0.05 \%)$ and once the skin scrapings were inoculated by using bacteriological loop/bent wire. Olive oil (Sterile) overlay was done as Malassezia furfur is a lipophilic yeast. Media were incubated in the room temperature up to 21 days/ three weeks and if there was no growth till then, it was considered as negative for Malassezia furfur. In most of the samples, yeasts grew by 5-7 days (95\%) except a few took 7-15 days $(5 \%)$.

The growth on SDA with was observed macroscopically and microscopically by Gram stain. Observed under 100X (Oil immersion microscope) for characteristic morphology like oval yeast like cells with unipolar budding which were Gram positive were considered as positive for culture.

Statistical analysis was made for $10 \% \mathrm{KOH}, 1 \%$ CSB and Culture and $\mathrm{P}$ value is found to be $\mathrm{P}<0.001$. 
CSB stain was from Hi Media Laboratories, Mumbai and $10 \% \mathrm{KOH}$ was from Merck.

All the authors have observed all the slides and cultures separately and recorded the findings and final diagnosis was made.

\section{Results}

Out of 120 samples, 80 samples were positive $(66.66 \%)$ and 40 samples were negative $(33.34 \%)$ for $10 \% \mathrm{KOH}$ mount, 112 were positive $(93.33 \%)$ and 08 were negative $(6.67 \%)$ for $1 \%$ Chicago Blue 6B stain and 116 samples yielded M.furfur on SDA with olive oil $(96.66 \%)$. 4 samples did not yield any growth on SDA with olive oil (3.34\%). Wet mounts were confirmed as positive by observing the characteristic morphology. This shows a 'banana and grapes' or 'spaghetti and meatballs' appearance. Our study could observe the same morphology in $10 \% \mathrm{KOH}$ mount (Pic.2) and in Chicago Blue stain mount (Pic.3,4) too.

From the colonies grown on SDA with olive oil, yeasts were identified as pinpoint to small, smooth creamy colonies. Microscopically after Gram stain: Gram positive yeasts, morphologically variable, occurring in spherical to oval, thick walled cells, budding from a narrow base.

Table 1 : Shows different investigations with results and percentage.

\begin{tabular}{|l|c|c|c|c|c|c|c|}
\hline & \multicolumn{2}{|c|}{$10 \% \mathrm{KOH}$} & \multicolumn{2}{c|}{ CSB stain } & \multicolumn{2}{c|}{ Culture } & Total \\
\cline { 2 - 8 } & Positive & Negative & Positive & Negative & Positive & Negative & \\
\hline No. of samples & 80 & 40 & 112 & 08 & 116 & 04 & 120 \\
\hline Percentage \% & 66.66 & 33.34 & 93.33 & 6.67 & 96.66 & 3.34 & 100 \\
\hline
\end{tabular}

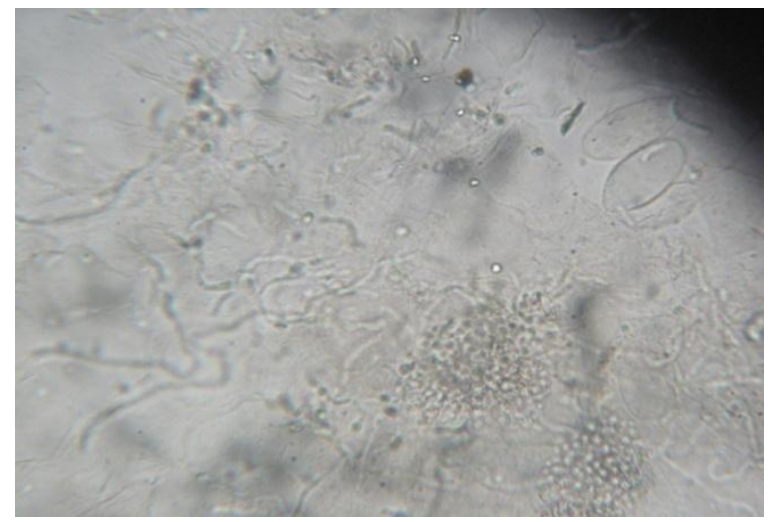

Photgraph 2: $10 \% \mathrm{KOH}$ mount showing fungal hyphae and spores.

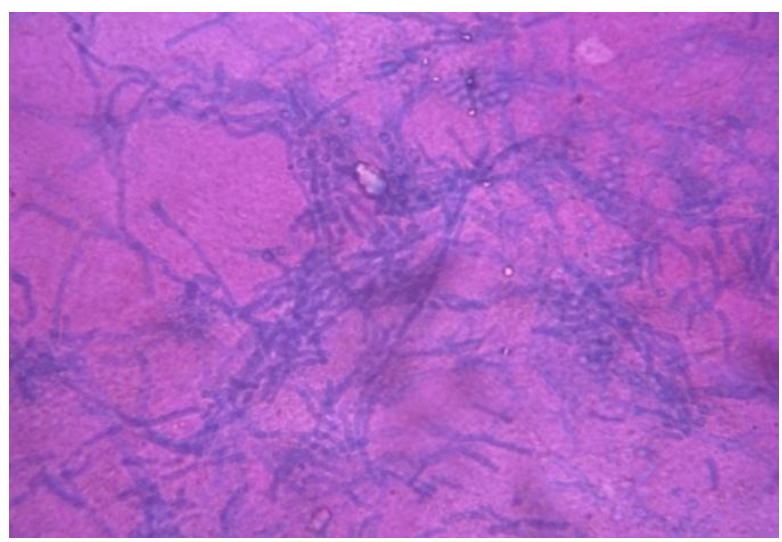

Photgraph 3: Photoghraphs showing blue colored hyphae and spores

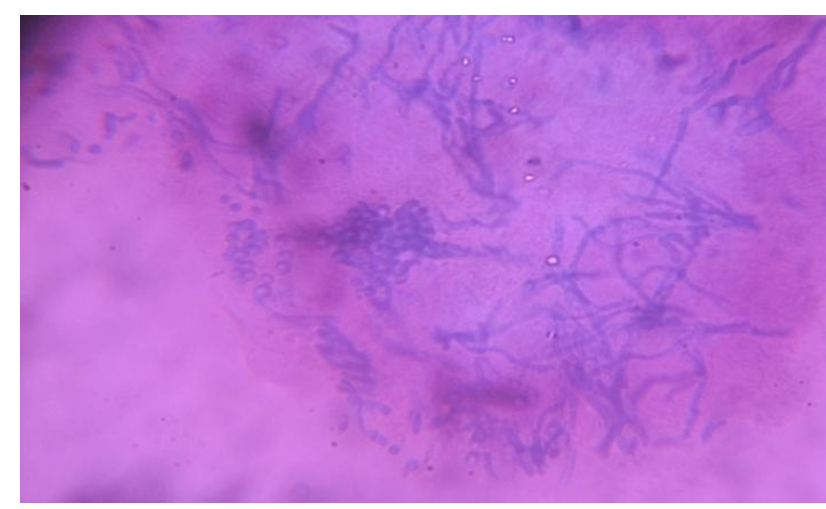

Photgraph 4: Photographs showing blue colored hyphae and spores as there is contrast with purple back ground and is a classical example of 'spaghetti and meat ball' appearance. 


\section{Conclusion}

With the above results $1 \%$ CSB stain can be incorporated as a rapid diagnostic tool for skin scrapings in all microbiology laboratories in suspected cases of Malassezia furfur. As a rapid test it saves time and as efficacy is better than just $10 \% \mathrm{KOH}$ mount this proves to be a superior technique and justifies the work. It is close to the Gold standard culture and provides a good color contrast too. Rapid diagnosis was possible with $1 \%$ CSB stain with a clear contrast.

Few researchers around the world have also published articles related to CSB stain and Dertmatophytosis, Candidiasis along with Malassezia furfur.

More dermatologists, clinicians, microbiologists start using this stain instead of mere $10 \% \mathrm{KOH}$ mount will prove to be encouraging them to diagnose rapidly with a contrast and more sensitive technique too.

\section{Future Aspects}

All the samples suspected to be of fungal origin should be subjected to $1 \%$ CSB stain. When more and more research work done and more papers published this will be useful for clinicians in the diagnosis of mycoses.

\section{References}

1. S.Fonseka, C.Lim, U.Bandara \& Manel D, New Contrast stain for the rapid diagnosis of Dermatophytosis and Pityriasis versicolor, Lab Medicine, Vol.42, Number 11, November 2011.pg 649-452

2. Tambosis E,Lim C, A comparison of the contrast stains ,Chicago Blue, Chlorazole black and Parker ink for the rapid diagnosis of skin and nail infections., Int $\mathbf{J}$ Dermatol, Aug 2012, 51(8); pg 935-938

3. Nitkita Lodha, Shital Amin Poojary, A novel contrast stain for the rapid diagnosis of Pityriasis versicolor : A comparison of Chicago sky Blue 6B stain, potassium hydroxide mount and culture. Ind Journal of Derma, vol 60,Iss. 4, 2015 pg 340-344.
4. Zhong Liu, Ping Sheng et al, Comparison of modified Chicago sky blue stain and potassium hydroxide mount for the diagnosis of dermatomycoses and onychomycoses., Journal of Microbial methods ,Vol.112,May 2105,pg 21-23

5. Lim CSH,Lim SL. New contrast stain for the rapid diagnosis of dermatophytic and candidal dermatomycosis. Arch Dermatol. 2008 Sep; 144(9); pg 1228-1229 